\title{
Multi-wavelength properties of AGN jets: some recent highlights
}

\section{Luigi Costamante*}

ASI - Italian Space Agency

Via del Politecnico snc, 00133 Rome, Italy

E-mail: luigi.costamantedasi.it

Relativistic jets from Active Galactic Nuclei (AGN) are powerful sources of radiation at every wavelength, but particularly in gamma-rays. Multi-wavelength observations in the last decade, driven by the new generation of large-area $\mathrm{GeV}$ and $\mathrm{TeV}$ telescopes, have revealed several new aspects of their structure and emission properties. This paper summarizes some recent progress in our understanding of relativistic jets, focusing in particular on the following results: a) evidence against the Broad Line Region (BLR) as origin of seed photons for the inverse Compton (IC) mechanism; b) spectral variability in gamma-rays that defies the usual SED classification, c) discovery of extreme-TeV sources, which challenge standard acceleration and emission scenarios, d) evidence of relativistic motion at the VLBI scale also in high-energy peaked BL Lacs. Other fundamental topics such as ultra-fast variability, neutrino emission and M87 images at event horizon scale are only briefly mentioned, as discussed in other contributions at these proceedings.

High Energy Phenomena in Relativistic Outflows VII - HEPRO VII

9-12 July 2019

Facultat de Fìsica, Universitat de Barcelona, Spain

\footnotetext{
* Speaker.
} 


\section{Introduction}

Relativistic jets in AGN are among the most powerful persistent phenomena in the Universe. They are strong emitters of radiation over the whole electromagnetic spectrum from radio to $\mathrm{TeV}$ energies, and dominate the extragalactic sky in gamma-rays. The 4th Fermi-LAT catalog of gamma-ray sources lists more than 3100 jetted AGN vs only 10 sources as normal/starburst galaxies [1]. Some of them could also be strong sources of high-energy neutrinos, as suggested by the marginally significant association (at the level of 3-3.5 $\sigma$ ) between the blazar TXS 0506+056 and a high-energy neutrino event and higher neutrino flux [2, 3, 4]. We learn about them, therefore, through multi-wavelength (and now multi-messanger) observations from both inside (Blazars) and outside (Radiogalaxies) their beaming cone $\sim 1 / \Gamma$ (where $\Gamma$ is the bulk-motion Lorentz factor).

We don't know yet the mechanism by which jets are launched, accelerated and collimated. The new results of the horizon telescope on M87 might yield some new clues in this respect. Their composition is uncertain as well, though most of their kinetic power required to feed the lobes must be provided by protons, otherwise the Compton drag on a pairs-dominated jet would effectively decelerate and stop the jet (e.g. ref. [5]). A composition of no more than $\sim 10$ pairs per proton seems the allowed range [6]. The two most likely sources of their power are the gravitational energy of the accreting matter or the rotational energy of the spinning black hole (BH). We now know that the former is not sufficient: at least part of the power must come from the BH spin, because the jet power derived from radiation (assuming 1 cold proton per emitting lepton) is often higher than the total accretion luminosity $\dot{M} c^{2}$ [7], in agreement with general relativistic MHD simulations [8, 9].

The spectral energy distribution (SED) of the jet emission, especially in blazars, is characterized by two broad humps, peaking at low and high energies. They are commonly (but not uniquely) explained as synchrotron and inverse Compton (IC) emission from a population of relativistic electrons. The origin of the seed photons for the IC scattering marks the difference among leptonic scenarios: synchrotron photons produced by the same electrons (Synchrotron Self-Compton mechanism, SSC) or by other parts of the jet (e.g. spine-layer/spine-sheath scenarios), or photons from radiation fields external to the jet (External Compton, EC), e.g. from the Broad Line region (BLR) or thermal infrared radiation from the dusty torus $[10,11]$.

Blazars can then be classified according to two main properties: the (non-thermal) jet emission and the (thermal) emission of the accretion flow. Jet-wise, blazars form a sequence of SEDs, with energies that go from radio to X-ray for the synchrotron peak and correspondingly from $\mathrm{MeV}$ to $\mathrm{TeV}$ for the gamma-ray peak. Blazars are thus divided in "Low-", "Intermediate-" and "HighSynchrotron Peaked" sources (LSP, ISP and HSP, see [12]) or "Low-" and "High-energy peaked" BL Lacs (LBL and HBL, see [13]). In the following I will use the terms HSP/HBL as equivalent. Thermally, blazars are divided into flat spectrum radio quasars (FSRQ) and BL Lac objects, according to the presence or absence of strong broad emission lines in their UV-optical spectra [14]. While there is a significant overlap between the two classes, and a percentage of sources are likely mis-classified due to the fact that the jet emission can swamp the thermal emission of the lines and the disk (so-called "masquerading BL Lacs" [15]), there is a real and substantial difference between FSRQ and BL Lacs: true BL Lacs have very weak or no line emission (with luminosities orders of magnitude lower than in FSRQ), weak or no torus emission and an underluminous accretion disk. This difference seems to occur at $L_{\text {disk }} / L_{E d d} \sim 10^{-2}$ [16], likely marking the transition between a 

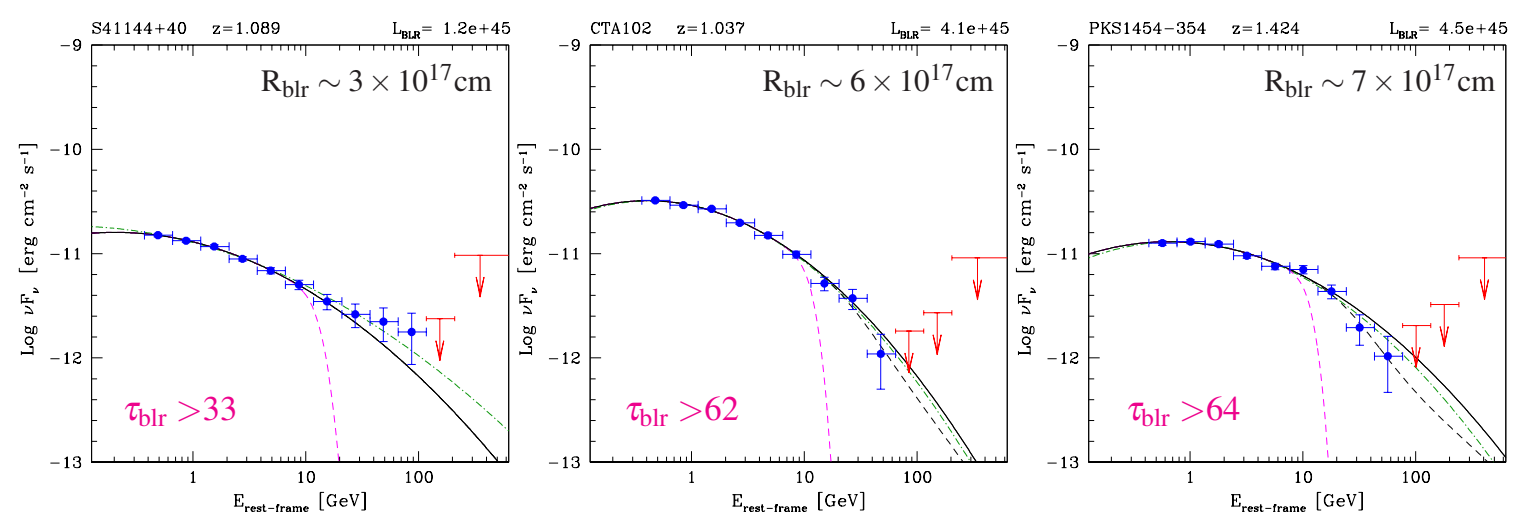

Figure 1: Test of the EC models on the gamma-ray SED of 3 FSRQ characterized by a large disk luminosity and BLR size, as labelled. Fermi-LAT spectra from 7.3 years of integration time. Upper limits at $2 \sigma$ level. Solid lines show the fit with parameters determined below $13 \mathrm{GeV}$ rest-frame. Black dashed lines show the same spectrum fitted with free BLR absorption, magenta lines the same spectrum with the optical depth expected if $R_{\mathrm{diss}}=R_{\mathrm{BLR}} / 2$, as labelled (see text). The $\tau_{b l r}$ values give the corresponding limits to the maximum optical depth, at the peak of the cross-section. Dot-dashed green lines show the fit of a pure log-parabolic model to the whole spectrum, without BLR absorption. The Fermi-LAT spectra do not show evidence of important $\gamma-\gamma$ absorption. From ref. [19].

radiatively efficient, geometrically thin, optically thick accretion flow to a radiatively inefficient, geometrically thick, optically thin accretion flow.

There is a connection between these two main properties: FSRQ are almost all LSP, are the most luminous and can have a large Compton dominance $\left(L_{I C} / L_{\text {synch }} \gtrsim 100\right)$, while BL Lacs span from LSP to HSP to "extreme" SEDs (where the synchotron emission peaks in the keV range). This has been interpreted as due to a different balance between acceleration and cooling of electrons [17, 18]: more powerful objects with luminous disks tend to have stronger cooling (by both synchrotron and IC) and thus have an SED which peaks at lower energies (LSP-type). When cooling is less (because of lower jet luminosity, lower magnetic field and absence of external radiation fields), the balance is reached progressively at higher energies and so the peaks shifts to higher frequencies (HSP-type SED).

While the new fundamental results of TXS 0506+056 as likely neutrino emitter and the first image of M87 with the Event Horizon Telescope are discussed in other contributions, here I will focus on some other new results.

\section{No EC with BLR photons}

The EC mechanism on BLR photons (i.e. an emitting zone inside the BLR) has been the most common scenario to model the SED of gamma-ray detected FSRQ (e.g. [17, 10, 18, 20]). It provides a straightforward way to explain both the difference in Compton dominance and SED peak frequencies between FSRQ and BLLac objects and the observed fast variability (few days to hours), indicative of compact emitting regions. However, those same external photons become targets for the $\gamma-\gamma$ collision and pair production process. The energy densities $U_{\text {rad }}$ used in EC models -calculated from the BLR-size/luminosity relation given by reverberation mapping- are large, of the order of $\sim 0.01 \mathrm{erg} \mathrm{cm}^{-3}$. The resulting optical depths become huge on photon paths 
as short as $10^{16} \mathrm{~cm}$. Thus we can expect on average a strong cut-off in the gamma-ray spectrum of EC-dominated FSRQ, appearing above $\sim 20 \mathrm{GeV}$ rest-frame and with maximum absorption at $\sim 100-200 \mathrm{GeV}$, corresponding to the peak of the $\gamma-\gamma$ cross section, precluding a VHE detection. In contrast, the VHE detections of the FSRQ 4C+21.35 [21], PKS 1510-08 [22, 23] and PKS $1441+25[24,25]$ indicated a gamma-ray emission coming from outside the BLR (there are now 8 FSRQ detected at VHE). However, these few detections didn't undermine the main paradigm for the FSRQ population, since in a distribution of locations and among objects with different BLR sizes, it could be expected that the tail could sometimes extend also beyond the BLR (e.g. in the internal shock scenario), especially during flares.

The Fermi-LAT all-sky survey provided the mean to test the EC paradigm on a much wider sample, once the exposure time was long enough to overcome the limited collection area $\left(\sim 1 \mathrm{~m}^{2}\right)$ and give meaningful limits beyond $10 \mathrm{GeV}$. This has been recently done on 7.3 years of data, checking the spectra of the 100 brightest FSRQ detected by Fermi-LAT [19], under the same assumptions and values adopted in the EC models with BLR photons [10,11]. The BLR spectrum can be approximated with a Planckian spectrum peaked at $10.2 \mathrm{eV}$, renormalized to match the BLR energy density. This provides a good approximation to the optical depth curve from the Hydrogen Ly $\alpha$ emission and recombination continuum complex, over a wide range of ionization states. The LAT spectrum is first fitted in the unabsorbed part (up to $13 \mathrm{GeV}$ rest-frame), with power-law and logparabolic models, and then extrapolated at higher energies, fitting the optical depth $\tau(E)=\ell \tau_{16}(E)$ to the data (where $\tau_{16}(E)$ is the optical depth for a path of $10^{16} \mathrm{~cm}$ ). Fits with both the unabsorbed spectrum fixed at the best-fit values and with all parameters free to vary were tested. The resulting photon path length $\ell$ and optical depth $\tau_{\max }$ are to be compared with the values from deep inside the BLR, $\ell_{\mathrm{BLR}}$ and $\tau_{\max }^{\mathrm{BLR}}$. As benchmark, one can assume that the dissipation region is located at $R_{\mathrm{BLR}} / 2$ (i.e. halfway between the BH and the BLR), with a lower limit of $2 \times 10^{16} \mathrm{~cm}$. Therefore $\tau_{\max }^{\mathrm{BLR}}$ is the optical depth corresponding to $\ell_{\mathrm{BLR}}=R_{\mathrm{BLR}}-R_{\mathrm{diss}}$.

Surprisingly, there is no evidence for the BLR cut-off, even in sources with large disk luminosities and BLR sizes (see Fig. 1). For $2 / 3$ of the sample, the LAT data exclude any significant absorption $\left(\tau_{\max }<1\right)$, while for the remaining $1 / 3$ the possible absorption is constrained to be $1.5-$ 2 orders of magnitude lower than expected (see Fig. 2). This result holds considering the emission from either a localized region inside the BLR or a single relativistic blob traveling over the whole BLR size. In the latter case, the observed gamma-rays are produced at different distances inside the BLR, with decreasing optical depth (since the photon path inside the BLR is decreasing). As a result, the time-integrated spectrum does not appear attenuated by $e^{-\tau(E, \ell)}$, but has a shallower shape which goes as $\tau^{-1}$ for large values of $\tau$. Even in this scenario however, the limits increase only mildly, by a factor 2-3. The large majority of objects still do not show significant absorption (see Fig. 2, magenta histogram). This result holds also dividing high and low-flux states, for the 20 brightest sources with sufficient statistics, and for powerful blazars with the largest BLR. Only one out of 10 objects seems compatible with substantial attenuation $\left(\tau_{\max }>5\right)$. For 9 out of 10 objects, the jet does not seem to interact with BLR photons for most of the time or of the flux, contrary to the EC model's assumption.

To keep a low $\tau$ inside the BLR, one can envisage two possibilities: 1) to decrease the photon densities by enlarging the size of the BLR (e.g. if the radius from reverberation mapping is underestimated), or 2) to shift the $\gamma-\gamma$ threshold at higher energies by selecting preferred angles of 

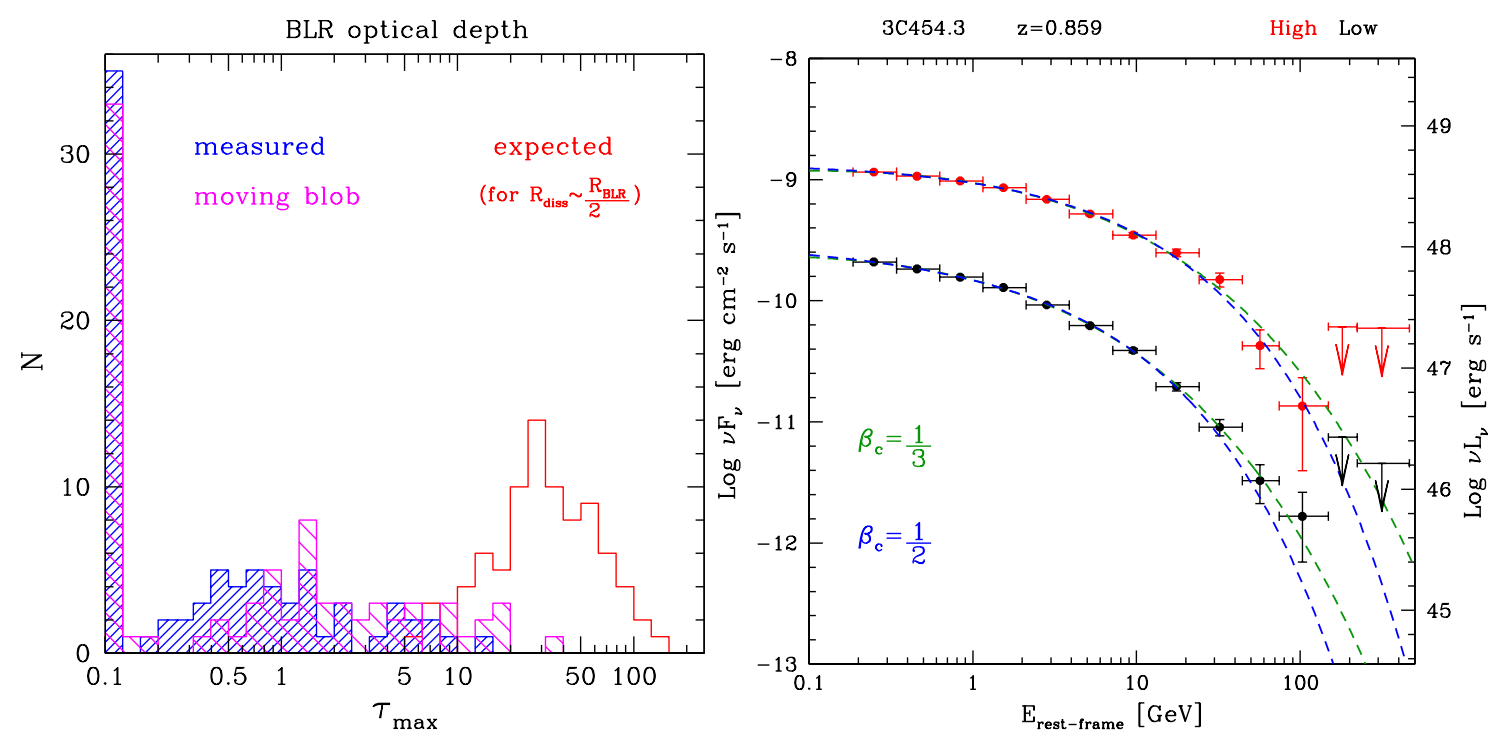

Figure 2: Left: histogram of the distribution of measured vs expected maximum optical depths $\tau_{\max }$ (i.e. at the peak of the $\gamma-\gamma$ cross-section), for the 83 objects with BLR luminosity estimates. Where $\tau_{\max } \leq 0.1$, objects are counted in the first bin. Three emission scenario are considered: from deep inside the BLR, as in EC models (red histogram), from a fixed location inside the BLR (blue histogram) and from a movingblog emitting up to the BLR radius (magenta histogram). The LAT data indicate that $\tau_{\max }$ due to BLR photons is at most $\sim 30-100 x$ lower than typically used in EC(BLR) models, and it is $<1$ in $\sim 2 / 3$ of the sample. Right: spectra of 3C 454.3 extracted in high and low state (namely, when the daily flux was above or below 1e- $\left.6 \mathrm{~cm}^{-2} \mathrm{~s}^{-1}\right)$. Total integration times are 148 and 1875 days, respectively. The function $N(E) \propto$ $E^{-\Gamma} \exp \left(-\left(E / E_{C}\right)^{\beta_{c}}\right)$ is fitted with two different exponents $\beta_{c}$, corresponding to IC with synchrotron $(1 / 3)$ or external Planckian photons (1/2). Details in text and refs [19, 26].

interaction. Both scenarios do not save the EC(BLR) model. In the first case, since $\tau \propto 1 / R_{\mathrm{BLR}}$, a factor $100 \times$ lower means that the size of the BLR should be $100 \times$ larger, in fact close to the torus size. This implies a factor $10^{-4}$ lower energy density for the BLR photons, which put them below all other available energy densities (e.g. by infrared photons or B). The second case can be realized if the BLR is flattened along the disk. To push the $20 \mathrm{GeV}$ absorption cut-off outside the observed data (e.g. at least $100 \mathrm{GeV}$ rest-frame), one needs an average shift of $\sim 5 \times$ of the energy threshold. Geometrical considerations show that the preferred angle should be $<30$ degrees, meaning that the region should be already outside the BLR radius. Both alternatives do not seem able to keep $\mathrm{EC}(\mathrm{BLR})$ as a viable mechanism for the gamma-ray emission in FSRQ.

This result has two direct consequences. The first is that VHE gamma-rays are not suppressed by the BLR photons. Therefore not only a few but the majority of FSRQ -even 3C 454.3- can be copious emitters of VHE gamma-rays under proper conditions, and could become easily detectable by Cherenkov telescopes depending on the particle spectrum, but especially during HBL-like flares (see next section). The second consequence is that the spectral shape of the radiation is not determined by absorption, but is intrinsic. This opens new diagnostic possibilities because there is a direct link between the distribution of parent electrons and the upscattered photons at the cut-off region [26]. An example is given by the spectra of 3C 454.3 (see Fig. 2). If the electron distribution is a power-law with an exponential cutoff of the form $\exp \left(-\left(E / E_{C}\right)^{2}\right)$, as expected when the cooling 
is $\propto \gamma^{2}$, the Thomson-upscattered gamma-ray spectrum will have a shape $\propto E^{-\Gamma} \exp \left(-\left(E / E_{C}\right)^{\beta_{c}}\right)$ with a smoother, under-exponential cut-off of $\beta_{c}=1 / 3$ or $1 / 2$, depending on the upscattered photon field, synchrotron or Planckian [26]. Indeed these shapes provide an excellent fit on the LAT data of 3C 454.3, in both high and low states, with a slight preference for a Planckian field in high state (maybe IR torus photons) and a SSC spectrum in low state (the other choices have a lower likelihood and probability of 0.11-0.16 under the Akaike information criterion for the goodness of fit). The comparison with the synchrotron spectrum could pin down the shape of the emitting particles at the highest energies (see also Zargaryan et al., these proceedings, and ref. [27]).

\section{HBL-like spectra in FSRQ}

On average, Fermi-LAT spectra of blazars follows the SED sequence as traced by the synchrotron peaks, shifting from steep $(\Gamma>2)$ to hard $(\Gamma<2)$ slopes as the SED changes from LSP to HSP [12]. However, several FSRQ and LSP sources have shown gamma-ray flares with hard LAT spectra, which are typical of HSP sources, without apparently changing their LSP character in the synchrotorn hump. Coupled with the absence of BLR absorption, the LAT spectra implies that FSRQ/LSP sources are capable of high VHE fluxes during such flares, even after the EBL attenuation, well within the sensitivity of present Cherenkov telescopes in just few hours [19]. Figure 3 shows 4 striking examples of these unexpected gamma-ray spectra in famous objects, 3 FSRQ (among which the neutrino source TXS 0506+056, an FSRQ in disguise [28]) and BL Lac itself.

The origin of these spectra is not yet clear: if it is a new component emerging only temporarily (sort of mini-HSP emitting zone inside a main LSP-type outflow), one would expect it to appear in the X-ray band as well, by synchrotron, in the form of a steep X-ray spectrum of higher flux. This seems not the case, requiring a huge Compton dominance of the new component to be consistent with the data. These HSP-like states have been reported so far only during active states, but that could be partly due to an observational bias.

One of these HSP-like states occurred during the neutrino flare of TXS 0506+056 (see Fig.3), but not around the IceCube-170922A event [31]. In the latter case the SED was LSP-like, both in $\mathrm{X}$-rays and gamma-rays, with no VHE emission. Note that the MAGIC detection occurred $\sim 10$ days after the neutrino event, in coincidence to a higher X-ray flux with steep spectrum (as observed by Swift, $\left.\Gamma_{X} \sim 2.5\right)$. In contrast, in the days of IceCube-170922A there are strong upper limits at VHE -about one order of magnitude lower than the detected flux- together with a low-flux X-ray spectrum with hard index, indicative of pure IC emission (as typical of LSP). In other words, the MAGIC detection seems most likely driven by an enhanced emission of high-energy electrons, as traced by their synchrotron emission entering the X-ray band (a common behaviour in ISP/HSP blazars). It cannot be directly related to the neutrino event [2], unless with a scenario explaining the 10-days delay (which becomes 100 days considering a beaming factor of 10).

\section{The Puzzle of Extreme-TeV BL Lacs}

Extreme-TeV BL Lacs are a new type of HBL characterized by a hard intrinsic VHE spectrum $\left(\Gamma_{\mathrm{VHE}} \lesssim 1.5-1.9\right)$, after correction for the effects of $\gamma-\gamma$ interactions with the diffuse Extragalactic Background Light (EBL, see e.g. [32]). This locates their gamma-ray peak in the SED above 2-10 

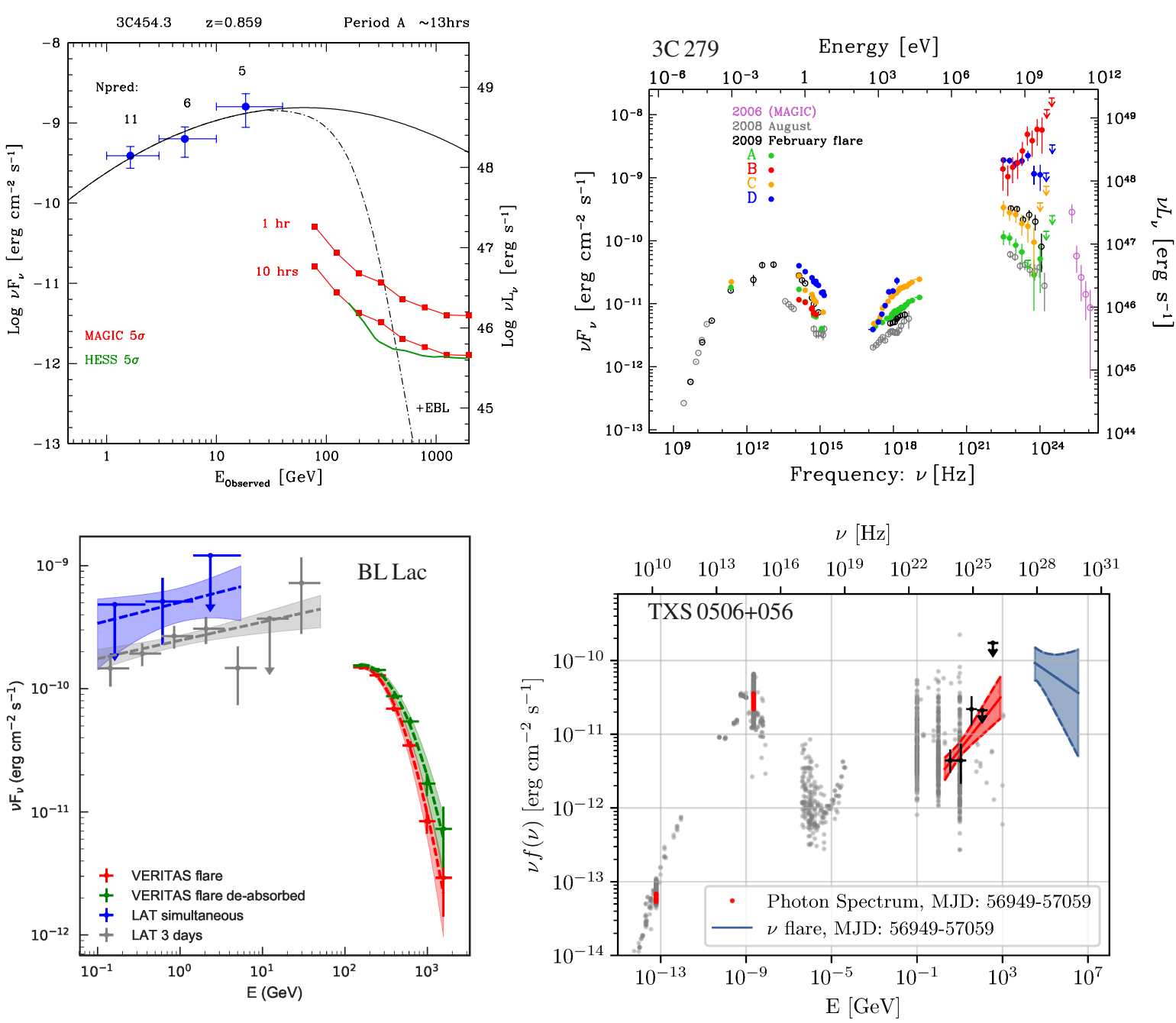

Figure 3: SED gamma-ray spectra of 4 non-HSP blazars of both FSRQ and BL Lac types, showing gammaray properties typical of HSP sources. Integration times range from few hours to several days. Observation dates for the plots on the left are Sept 9, 2013 (3C 454.3) and Oct 5, 2016 (BL Lac). The 3C 454.3 plot shows also the sensitivity curves of present Cherenkov telescopes. From refs [19, 29, 30, 31].

$\mathrm{TeV}$, the highest peak energies ever seen in blazars and 1-2 orders of magnitude higher than regular HSP/HBL objects (see Fig. 4). A hard spectrum at such high energies is difficult to obtain by IC in blazars, for standard one-zone leptonic models of the SED. Both the decrease of the scattering efficiency in the Klein-Nishina regime and the lower energy density of the seed photons available for scatterings in the Thomson regime, as the gamma-ray energy increases, tend to steepen the $\mathrm{TeV}$ spectrum. So far, they seem to constitute about 1/4 of all HBLs detected at VHE [33]. By synchrotron, most (but not all) of them are also of the extreme type, i.e. with the synchrotron peak above $1 \mathrm{keV}$ up to $100 \mathrm{keV}$ and beyond. However, even such high observed synchrotron frequencies are well below the maximum frequency that can be produced by electrons shock-accelerated at the maximum possible rate $\left(h v_{\text {peak }} / \delta \sim 1-10 \mathrm{KeV} \ll 150 \mathrm{MeV}, \delta\right.$ being the beaming factor). Although extreme, even these blazars are still not extreme accelerators.

Such hard TeV spectra could be the result of a new narrow-peaked electron population, whose 


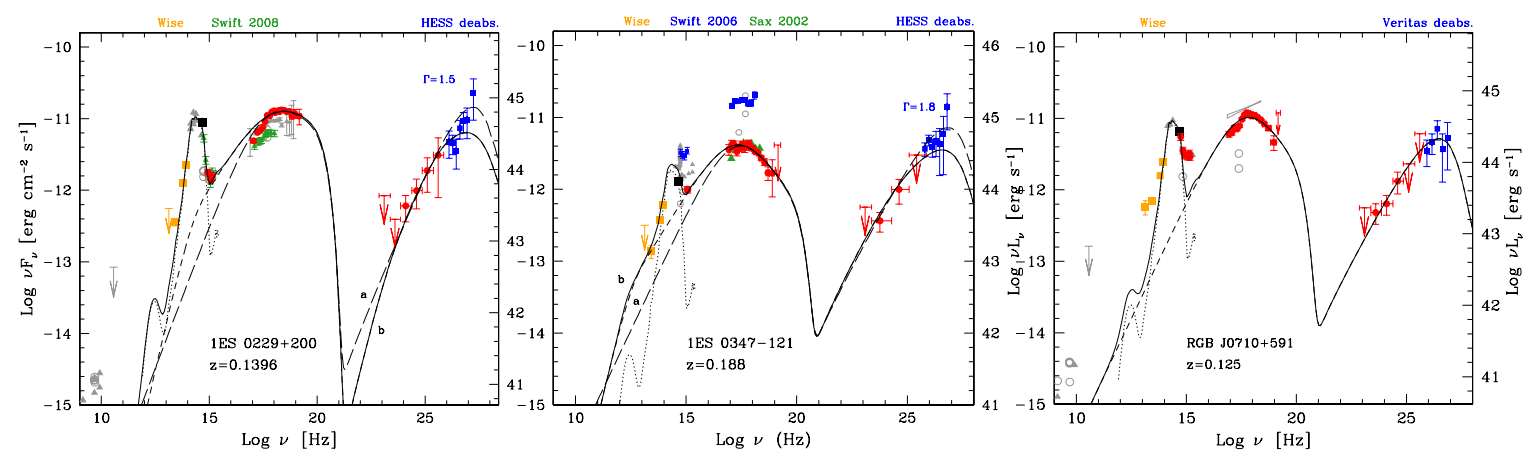

Figure 4: SEDs of 3 hard-TeV BL Lacs, with new simultaneous Swift-NuSTAR-Fermi data shown in red. Historical data taken close to the same epoch of the VHE data are shown in blue. The VHE data are corrected for EBL absorption following [37]. Solid lines show the sum of the theoretical SSC model (short-dashed line) and host-galaxy emission (dotted line). From ref. [34].

synchrotron emission should emerge in hard X-rays. This seems excluded by recent NuSTARSwift observations, which pinned down the spectrum of the synchrotron hump for six objects, from UV to hard X-rays [34]. A one-zone SSC model could in principle reproduce the extreme properties of both peaks in the SED, from X-ray up to TeV energies. However, it requires 1) high peak-electrons energies $\left(\gamma \gtrsim 10^{6}\right)$ with extremely low radiative efficiency, 2) milliGauss magnetic fields and conditions heavily out of equipartition (by 3 to 5 orders of magnitude), and 3) not fitting the simultaneous UV data, which then should belong to a different emission component, possibly the same as the far-IR (WISE) data [34]. There is indeed evidence in two objects of a separation between the UV and X-ray emission (see Fig. 4), with the X-ray power-law spectrum underestimating the UV flux when extrapolated at lower energies. This has also been reported lately for Mkn 501 during a high and extreme -but temporary-state in 2012 [35].

Whatever scenario is considered, radiative cooling must be inhibited: the $\mathrm{TeV}$ electrons cannot "see" UV or lower-energy photons, even if coming from different zones/populations, otherwise the increased radiative cooling in Thomson regime would steepen the VHE spectrum. This implies that even the assumption of a structured jet -namely a fast spine surrounded by a slower layer- would not help in reaching equipartition.

Conditions so far away from equipartition are puzzling also because not limited to a flaring episode: the extreme-TeV spectra in most of these BL Lacs seem to last for years. There must be a mechanism which keep the conditions persistently out of equipartition. The high values of the ratio of particles to magnetic field energy density $\left(U_{\mathrm{e}} / U_{\mathrm{B}} \sim 10^{3}-10^{5}\right)$ seems also to exclude magnetic reconnection as acceleration mechanism. Models predict an upper limit of the order of $U_{\mathrm{e}} / U_{\mathrm{B}} \sim 3$ in the dissipation region [36]. These blazars might be revealing something new of the different processes at work in relativistic jets.

\section{Ubiquitous Ultra-fast Variability}

Ultra-fast variability is a surprising new aspect of AGN jets discovered in the last decade, as soon as large-area gamma-ray detectors became operational. It can be defined as a variability time which is comparable or less than the light-crossing time of the putative Black Hole (e.g. $\tau_{0} \equiv R_{S} / c$ ). It is surprising because it apparently defies the paradigm that the minimum variability 
in the jet would be imprinted by the scale of the central BH horizon (relativistic beaming cannot shorten the variability timescale imprinted by a source that is stationary in the observer's frame, as remarked by ref [38]). It thus indicates that the observed variability is imprinted either by a fraction of the BH horizon, or by small-scale fluctuations intrinsic to the jet itself [38]. The most striking example is provided by the huge flare of PKS 2155-304, with doubling timescales $<2$ minutes corresponding to $\sim 0.01 \tau_{0}$, moreover at a flux scale $10-100 \times$ higher than normal [39].

This topic is fully addressed in Maxim Barkov's contribution (these proceedings), here I only point out that such phenomenon is now observed in every type of jetted AGNs. It is observed in every class of blazars, be it BL Lacs of HSP type as PKS 2155-304 [39] and Mkn 501 [40] or LSP-type as BL Lac itself [30], or FSRQ as 3C 279 in 2015 [29] and 4C 21.35 [21] (the latter at VHE, which is significant since it indicates an extremely compact region outside the BLR). It is also observed in slightly misaligned jets, like the radiogalaxies M87 [41, 42] and IC 310 [43, 44]. In the latter case, a luminosity higher than the upper limit of $2 \times 10^{43} \mathrm{erg} \mathrm{s}^{-1}$ for 5-minute flares seems to exclude a BH magnetosphere origin of the flare detected from IC 310 [45].

Though timescales can vary from minutes to days depending on the different beaming factors and region sizes, it seems that such phenomenon is a fundamental feature of relativistic jets, and it is revealed only in gamma-rays: there is no report or clear evidence so far for such variability in the synchrotron part of the emission corresponding to the gamma-ray flares, namely X-rays for VHE gamma-rays or optical for $\mathrm{GeV}$ emission.

\section{Superluminal Motion}

One of the long-standing problems in blazar physics is the apparent discrepancy between the high bulk-motion Lorentz factors required by gamma-ray transparency and SED modeling $(\Gamma \sim$ $10-50$ ) and the absence of significant superluminal motion at the VLBI scale (so-called "bulk Lorentz factor crisis"). While FSRQ and LSP-type blazars show high apparent jet speed, up to 3040, the VLBI radio knots seem stationary or at most subluminal in HSP-type BL Lacs [46]. This is particularly evident in Mkn 421, for example on the results of the MOJAVE survey [47]. Absence of superluminal motion is expected for very small viewing angles, but that hypothesis would make the parent population numbers larger than the known radio-loud population.

So far it has been generally assumed that the jet was partially disrupted or stopped before the VLBI scale, and/or that the VLBI knots were the location of standing recollimation shocks accelerating particles along the jet. Evidence for the latter is possibly emerging now [47]. Using 13 years of observations with Swift and stacking the X-ray lightcurves of 6 different active periods in Mkn 421, a common variability pattern seems to emerge (see Fig. 5). It is consistent with a main flare emission zone located in the most upstream $15.3 \mathrm{GHz}$ radio knot, at 0.38 mas from the core. Subsequent peaks in the lightcurve are then consistent with a perturbation crossing all the downstream radio knots with a constant apparent speed $\beta=45 c$. The significance of the observed pattern not arising from stochastic processes is found above $3 \sigma$ [47].

With the caveats of the choice of which radio knot is important and their possible movement in time (the average radio positons are derived from observations in 2006 to 2013 , while the X-ray flare data refer to the period 2013-2018), this result seems to indicate that the jet is indeed highly relativistic at the VLBI scale, in HSP as well as FSRQ blazars. If true, it would also confirm that 

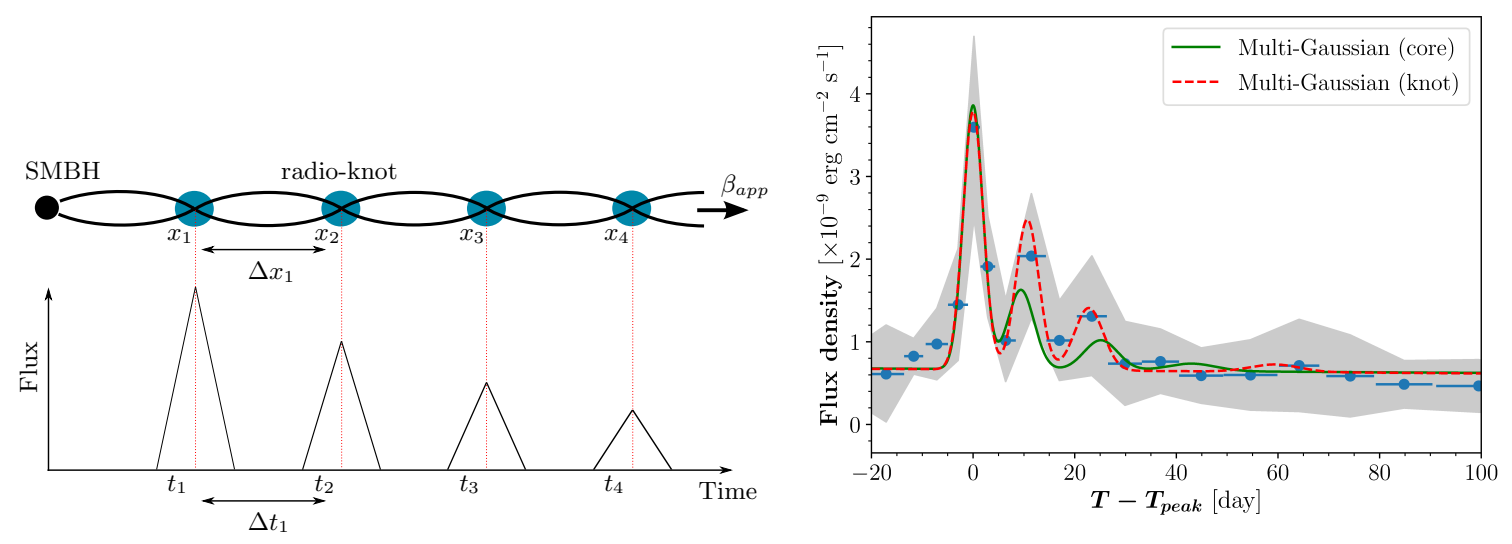

Figure 5: Left: scheme of the expected lightcurve signature of a perturbation traveling across knots $x_{i}$ with apparent speed $\beta_{a p p}$ and delay $\Delta t_{1}$ between two consecutive flares. Right: flare models of the stacked X-ray lightcurves, starting from a main flare in the radio core. From ref. [47].

the dissipation zones along the jet are much more stationary at certain distances from the BH than in FSRQ, denoting another essential difference in jet structure between these two blazar classes.

\section{Conclusions}

Observations allowed by a new generation of large-area gamma-ray telescopes (Cherenkov telescopes arrays and Fermi-LAT) have unveiled new aspects of relativistic jets in AGNs, driving the efforts for a more complete multi-wavelength picture of their properties. On one hand, FermiLAT data are showing that BLR photons are not the main seed photons for the IC mechanism, undermining the main explanation of the difference in SED properties between FSRQ and BL Lacs. A straightforward alternative for the EC mechanism is given by the IR photons from the torus [48, 11], though this implies a change of the derived jet parameters. Another possibility is provided by other photon fields from different parts of the jet (or via the SSC mechanism itself), but in this case the difference in thermal properties between FSRQ and BL Lacs acts just as a proxy for a structural difference in jet launching and power, and does not influence directly the cooling of jet particles. The HBL-behaviour of some FSRQ in gamma-rays is further blurring the difference with BL Lacs. Studies of close-by off-axis jets (as in the radiogalaxies M87, CenA, IC 310) might give new insights, as well as further investigation of the ultra-fast variability.

On the other hand, the discovery of extreme-TeV BL Lacs is challenging also the SSC paradigm. The parameters for it to work seem somewhat unrealistic ( $\mathrm{mG}$ magnetic fields, pile-up electrons distributions, steadily out of equipartition by 5 orders of magnitude) and the necessity of multiple ad-hoc components to explain the SED is detrimental for the elegance and simplicity of the original concept. The gamma-ray emission might be related to a different process. It still remains a puzzle that, even in the most extreme cases, the acceleration rate in blazars' jets seems orders of magnitude less than in the Crab or SNRs.

Acknowledgement: I am grateful to the organisers for the invitation to such enjoyable conference.

\section{References}

[1] The Fermi-LAT collaboration, Fermi Large Area Telescope Fourth Source Catalog, ArXiv e-prints 
(2019) [1902.10045].

[2] IceCube Collaboration, M. G. Aartsen, M. Ackermann, J. Adams, J. A. Aguilar, M. Ahlers et al., Multimessenger observations of a flaring blazar coincident with high-energy neutrino IceCube-170922A, Science 361 (2018) eaat1378 [1807.08816].

[3] IceCube Collaboration, M. G. Aartsen, M. Ackermann, J. Adams, J. A. Aguilar, M. Ahlers et al., Neutrino emission from the direction of the blazar TXS 0506+056 prior to the IceCube-170922A alert, Science 361 (2018) 147 [1807.08794].

[4] F. Halzen, Cosmic Neutrinos and the Cosmic-Ray Accelerator TXS 0506+056, arXiv e-prints (2019) arXiv:1909.09468 [1909.09468].

[5] M. Sikora and G. Madejski, On Pair Content and Variability of Subparsec Jets in Quasars, ApJ 534 (2000) 109 [astro-ph/9912335].

[6] G. Ghisellini and F. Tavecchio, Compton rockets and the minimum power of relativistic jets, MNRAS 409 (2010) L79 [1008.1982].

[7] G. Ghisellini, F. Tavecchio, L. Maraschi, A. Celotti and T. Sbarrato, The power of relativistic jets is larger than the luminosity of their accretion disks, Nature 515 (2014) 376 [1411.5368].

[8] R. Narayan, Y. Zhu, D. Psaltis and A. Sal̆e11owski, HEROIC: 3D general relativistic radiative post-processor with comptonization for black hole accretion discs, MNRAS 457 (2016) 608 [1510.04208].

[9] A. Tchekhovskoy, R. Narayan and J. C. McKinney, Efficient generation of jets from magnetically arrested accretion on a rapidly spinning black hole, MNRAS 418 (2011) L79 [11 08 . 0412 ].

[10] G. Ghisellini and F. Tavecchio, Canonical high-power blazars, MNRAS 397 (2009) 985 [0902.0793].

[11] M. Sikora, Ł. Stawarz, R. Moderski, K. Nalewajko and G. M. Madejski, Constraining Emission Models of Luminous Blazar Sources, ApJ 704 (2009) 38 [0904 . 1414 ].

[12] M. Ackermann, M. Ajello, W. B. Atwood, L. Baldini, J. Ballet, G. Barbiellini et al., The Third Catalog of Active Galactic Nuclei Detected by the Fermi Large Area Telescope, ApJ 810 (2015) 14 [1501.06054].

[13] P. Padovani and P. Giommi, The connection between $x$-ray-and radio-selected BL Lacertae objects, ApJ 444 (1995) 567 [astro-ph/9412073].

[14] C. M. Urry and P. Padovani, Unified Schemes for Radio-Loud Active Galactic Nuclei, PASP 107 (1995) 803 [astro-ph/9506063].

[15] P. Giommi, P. Padovani and G. Polenta, A simplified view of blazars: the $\gamma$-ray case, MNRAS 431 (2013) 1914 [1302.4331].

[16] T. Sbarrato, P. Padovani and G. Ghisellini, The jet-disc connection in AGN, MNRAS 445 (2014) 81 [1405.4865].

[17] G. Ghisellini, A. Celotti, G. Fossati, L. Maraschi and A. Comastri, A theoretical unifying scheme for gamma-ray bright blazars, MNRAS 301 (1998) 451 [astro-ph/9807317].

[18] G. Ghisellini and F. Tavecchio, The blazar sequence: a new perspective, MNRAS 387 (2008) 1669 [0802.1918].

[19] L. Costamante, S. Cutini, G. Tosti, E. Antolini and A. Tramacere, On the origin of gamma-rays in Fermi blazars: beyond the broad-line region, MNRAS 477 (2018) 4749 [1804.02408]. 
[20] J. D. Finke, Compton Dominance and the Blazar Sequence, ApJ 763 (2013) 134 [1212 . 0869 ].

[21] J. Aleksić, L. A. Antonelli, P. Antoranz, M. Backes, J. A. Barrio, D. Bastieri et al., MAGIC Discovery of Very High Energy Emission from the FSRQ PKS 1222+21, ApJ 730 (2011) L8 [1101. 4645$].$

[22] A. Abramowski, F. Acero, F. Aharonian, A. G. Akhperjanian, G. Anton, S. Balenderan et al., H.E.S.S. discovery of VHE $\gamma$-rays from the quasar PKS 1510-089, A\&A 554 (2013) A107 [1304.8071].

[23] J. Aleksić, S. Ansoldi, L. A. Antonelli, P. Antoranz, A. Babic, P. Bangale et al., MAGIC gamma-ray and multi-frequency observations of flat spectrum radio quasar PKS 1510-089 in early 2012, A\&A 569 (2014) A46 [1401.5646].

[24] A. U. Abeysekara, S. Archambault, A. Archer, T. Aune, A. Barnacka, W. Benbow et al., Gamma-Rays from the Quasar PKS 1441+25: Story of an Escape, ApJ 815 (2015) L22 [1512 . 04434 ].

[25] M. L. Ahnen, S. Ansoldi, L. A. Antonelli, P. Antoranz, A. Babic, B. Banerjee et al., Very High Energy $\gamma$-Rays from the Universe's Middle Age: Detection of the $z=0.940$ Blazar PKS 1441+25 with MAGIC, ApJ 815 (2015) L23 [1512. 04435$].$

[26] E. Lefa, S. R. Kelner and F. A. Aharonian, On the Spectral Shape of Radiation due to Inverse Compton Scattering Close to the Maximum Cutoff, ApJ 753 (2012) 176 [120 5 . 2929].

[27] C. Romoli, A. M. Taylor and F. Aharonian, Cut-off characterisation of energy spectra of bright fermi sources: Current instrument limits and future possibilities, Astroparticle Physics 88 (2017) 38 [1608.01501].

[28] P. Padovani, F. Oikonomou, M. Petropoulou, P. Giommi and E. Resconi, TXS 0506+056, the first cosmic neutrino source, is not a BL Lac, MNRAS 484 (2019) L104 [1901 . 06998 ].

[29] M. Hayashida, K. Nalewajko, G. M. Madejski, M. Sikora, R. Itoh, M. Ajello et al., Rapid Variability of Blazar 3C 279 during Flaring States in 2013-2014 with Joint Fermi-LAT, NuSTAR, Swift, and Ground-Based Multiwavelength Observations, ApJ 807 (2015) 79 [1502. 04699 ].

[30] A. U. Abeysekara, W. Benbow, R. Bird, T. Brantseg, R. Brose, M. Buchovecky et al., Multiwavelength Observations of the Blazar BL Lacertae: A New Fast TeV Gamma-Ray Flare, ApJ 856 (2018) 95 [1802.10113].

[31] P. Padovani, P. Giommi, E. Resconi, T. Glauch, B. Arsioli, N. Sahakyan et al., Dissecting the region around IceCube-170922A: the blazar TXS 0506+056 as the first cosmic neutrino source, MNRAS 480 (2018) 192 [1807.04461].

[32] L. Costamante, Gamma-Rays from Blazars and the Extragalactic Background Light, International Journal of Modern Physics D 22 (2013) 1330025 [1309. 0612].

[33] L. Costamante, TeV-peaked candidate BL Lac objects, arXiv e-prints (2019) arXiv:1911.05027 [1911.05027].

[34] L. Costamante, G. Bonnoli, F. Tavecchio, G. Ghisellini, G. Tagliaferri and D. Khangulyan, The NuSTAR view on hard-TeV BL Lacs, MNRAS 477 (2018) 4257 [1711. 06282].

[35] M. L. Ahnen, S. Ansoldi, L. A. Antonelli, C. Arcaro, A. Babić, B. Banerjee et al., Extreme HBL behavior of Markarian 501 during 2012, A\&A 620 (2018) A181 [1808. 04300 ].

[36] L. Sironi, M. Petropoulou and D. Giannios, Relativistic jets shine through shocks or magnetic reconnection?, MNRAS 450 (2015) 183 [1502.01021].

[37] A. Franceschini, G. Rodighiero and M. Vaccari, Extragalactic optical-infrared background radiation, its time evolution and the cosmic photon-photon opacity, A\&A 487 (2008) 837. 
[38] M. C. Begelman, A. C. Fabian and M. J. Rees, Implications of very rapid TeV variability in blazars, MNRAS 384 (2008) L19 [0 709.0540$].$

[39] F. Aharonian, A. G. Akhperjanian, A. R. Bazer-Bachi, B. Behera, M. Beilicke, W. Benbow et al., An Exceptional Very High Energy Gamma-Ray Flare of PKS 2155-304, ApJ 664 (2007) L71 [0706.0797].

[40] J. Albert, E. Aliu, H. Anderhub, P. Antoranz, A. Armada, C. Baixeras et al., Variable Very High Energy $\gamma$-Ray Emission from Markarian 501, ApJ 669 (2007) 862 [astro-ph/ 0702008 ].

[41] F. Aharonian, A. G. Akhperjanian, A. R. Bazer-Bachi, M. Beilicke, W. Benbow, D. Berge et al., Fast Variability of Tera-Electron Volt $\gamma$ Rays from the Radio Galaxy M87, Science 314 (2006) 1424 [astro-ph/0612016].

[42] V. A. Acciari, E. Aliu, T. Arlen, M. Bautista, M. Beilicke, W. Benbow et al., Radio Imaging of the Very-High-Energy $\gamma$-Ray Emission Region in the Central Engine of a Radio Galaxy, Science 325 (2009) 444 [0908.0511].

[43] J. Aleksić, S. Ansoldi, L. A. Antonelli, P. Antoranz, A. Babic, P. Bangale et al., Black hole lightning due to particle acceleration at subhorizon scales, Science 346 (2014) 1080 [1412 . 4936].

[44] M. Kadler, D. Eisenacher, E. Ros, K. Mannheim, D. Elsässer and U. Bach, The blazar-like radio structure of the TeV source IC 310, A\&A 538 (2012) L1 [1201.2870].

[45] F. A. Aharonian, M. V. Barkov and D. Khangulyan, Scenarios for Ultrafast Gamma-Ray Variability in AGN, ApJ 841 (2017) 61 [1704.08148].

[46] M. L. Lister, D. C. Homan, T. Hovatta, K. I. Kellermann, S. Kiehlmann, Y. Y. Kovalev et al., MOJAVE. XVII. Jet Kinematics and Parent Population Properties of Relativistically Beamed Radio-loud Blazars, ApJ 874 (2019) 43 [1902 . 09591].

[47] O. Hervet, D. A. Williams, A. D. Falcone and A. Kaur, Probing an X-Ray Flare Pattern in Mrk 421 Induced by Multiple Stationary Shocks: A Solution to the Bulk Lorentz Factor Crisis, ApJ 877 (2019) $26[1904.06802]$.

[48] M. Sikora, M. C. Begelman and M. J. Rees, Comptonization of diffuse ambient radiation by a relativistic jet: The source of gamma rays from blazars?, ApJ 421 (1994) 153. 